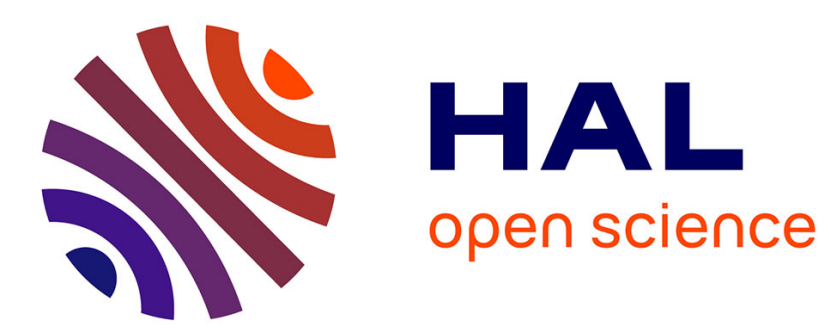

\title{
Precision of the IAS monitoring system based on the elapsed time method in the spectral domain
}

Hugo Andre, François Girardin, A. Bourdon, Jérôme Antoni, Didier Rémond

\section{To cite this version:}

Hugo Andre, François Girardin, A. Bourdon, Jérôme Antoni, Didier Rémond. Precision of the IAS monitoring system based on the elapsed time method in the spectral domain. Mechanical Systems and Signal Processing, 2014, 44 (1-2), pp.14-30. 10.1016/j.ymssp.2013.06.020 . hal-00916257

\section{HAL Id: hal-00916257 \\ https://hal.science/hal-00916257}

Submitted on 1 Jun 2019

HAL is a multi-disciplinary open access archive for the deposit and dissemination of scientific research documents, whether they are published or not. The documents may come from teaching and research institutions in France or abroad, or from public or private research centers.
L'archive ouverte pluridisciplinaire HAL, est destinée au dépôt et à la diffusion de documents scientifiques de niveau recherche, publiés ou non, émanant des établissements d'enseignement et de recherche français ou étrangers, des laboratoires publics ou privés. 
Systems and Signal Processing $\quad$ Manuscript Draft

Manuscript Number:

Title: Precision of the IAS Monitoring System based on the Elapsed Time method in the spectral domain.

Article Type: Special Iss: Instantaneous Angular Speed

Keywords: Instantaneous Angular Speed; Angular Sampling; Aliasing;

Quantization Precision

Corresponding Author: Mr francois Girardin, Ph.D.

Corresponding Author's Institution: Femto-st / ENSMM

First Author: Hugo André, Ph.D

Order of Authors: Hugo André, Ph.D; francois Girardin, Ph.D.; Adeline Bourdon; Jérome Antoni; Didier Rémond

Abstract: Instantaneous Angular speed (IAS) has recently appeared as an original and promising tool for monitoring mechanical parts of rotating machines. Mechanisms running under non stationary conditions, such as wind turbine, are especially suited for this method since the issued signal is intrinsically sampled in the angular domain. Although processing tools are developed to enhance its use in the industry, this method is lacking a proper identification of its limitations and this paper aims at precisely understanding two of its main shortcomings: the aliasing and the quantization phenomena. After having presented the measurement method, both the aliasing and the quantization error are theoretically dissected. Formula are proposed to estimate their influence in the spectral observation of the IAS, and a good Signal-to-Noise ratio appraisal for this measurement technique is finally obtained. It can eventually be used as a first guide to conveniently design an IAS based control / monitoring system. 


\section{*Covering Letter}

Dear Journal Editor

Please, find in attached files our contribution entitled "Precision of the IAS Monitoring System based on the Elapsed Time method in the spectral domain." (Authors: H. André, F. Girardin, A. Bourdon, J. Antoni, D. Rémond). It concerns major considerations about angular speed measurement and its spectral observations. Please accept it as a candidate for publication in your "Mechanical Systems and Signal Processing" journal, for the special issue on instantaneous angular speed and angular applications.

This paper is our original unpublished work and it has not been submitted to any other journal for reviewing. If you have any questions, please feel free to contact us. Any suggestion would be more than welcome. Thank you very much for your time and concern.

Sincerly,

The authors 
Highlights

- Instantaneous Angular Speed measurement using Elapsed Time method is studied

- Elapsed Time method is shown to induce low-pass filter on IAS spectrum.

- Aliasing is attenuated by the cardinal sine shape of the filter induced.

- Quantization errors and Signal-to-noise ratio are determined.

- Results can be used to conveniently design an IAS monitoring system. 


\title{
Precision of the IAS Monitoring System based on the Elapsed Time method in the spectral domain.
}

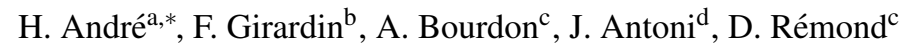 \\ ${ }^{a}$ Maia Eolis, Tour de Lille, Boulevard de Turin, Lille, 59000, France \\ ${ }^{b}$ FEMTO-ST, ENSMM, rue de l'Epitaphe, Besancon, F-25000, France. \\ ${ }^{c}$ LaMCoS, INSA-Lyon, University of Lyon, 18 rue des Sciences, Villeurbanne, F-69621, France \\ ${ }^{d}$ LVA, INSA-Lyon, University of Lyon, 25 bis avenue Jean Capelle, Villeurbanne, F-69621, France
}

\begin{abstract}
Instantaneous Angular Speed (IAS) has recently appeared as an original and promising tool for monitoring mechanical parts of rotating machines. Mechanisms running under non stationary conditions, such as wind turbine, are especially suited to this method since the issued signal is intrinsically sampled in the angular domain. Although processing tools are developed to enhance its use in the industry, this method is lacking a proper identification of its limitations and this paper aims at precisely understanding two of its main shortcomings: the aliasing and the quantization phenomena. After having presented the measurement method, both the aliasing and the quantization error are theoretically dissected. Formula are proposed to estimate their influence in the spectral observation of the IAS, and a good Signalto-Noise ratio appraisal for this measurement technique is finally obtained. It can eventually be used as a first guide to conveniently design an IAS based control / monitoring system.
\end{abstract}

Keywords: Instantaneous Angular Speed, Angular Sampling, Aliasing, Quantization Precision

\footnotetext{
IAS: Instantaneous Angular Speed

* Corresponding author

Email addresses: handre@maiaeolis.fr(H. André), francois.girardin@insa-lyon. fr (F. Girardin), adeline.bourdon@insa-lyon.fr(A. Bourdon), jerome.antoni@insa-lyon.fr(J. Antoni), didier.remondeinsa-lyon.fr(D. Rémond) 


\title{
Nomenclature
}

\author{
IAS Instantaneous Angular Speed \\ $F_{c} \quad$ Zero crossing detection clock speed \\ $T_{c}=1 / F_{c} \quad$ Time separating two counter pulses \\ $R \quad$ Resolution of the encoder, number of encoder segments \\ $N \quad$ Length of the measurement (number of points) \\ $f_{\theta} \quad$ Cyclic frequency $\left(\mathrm{rad}^{-1}\right)$ \\ $\theta_{i} \quad$ Encoder segments angular position (starting from $\theta_{0}$ at the beginning of the measurement) \\ $\Delta \theta=2 \pi / R \quad$ Angular increment between two segments of the encoder \\ $t_{i}=t\left(\theta_{i}\right) \quad$ Actual time for the $i^{t h}$ segment \\ $\tau_{i}=\tau\left(\theta_{i}\right) \quad$ Actual time difference between the segments respectively labelled $i$ and $i-1$ \\ $\omega_{i}=\omega\left(\theta_{i}\right) \quad$ Actual IAS at the exact position $\theta_{i}$ \\ $\bar{\omega}(\theta)$ \\ $\hat{t}_{i}, \hat{\tau}_{i}, \hat{\omega}_{i}$ \\ $X(\theta, \xi)$ \\ Actual IAS issued from moving average along $\Delta \theta$ : virtual signal to be sampled by E.T. measurement \\ Measured time, measured time difference and measured angular speed \\ Corresponds to a set of sequence of the discrete variable $\theta$, the set being indexed by the variable $\xi$ \\ A random signal is a bivariate quantity, depending both on the angular position $\theta$ and the trial $\xi$ \\ $X\left(\theta, \xi_{i}\right)=X_{i}(\theta) \quad$ Random process realisation obtained once the trial is fixed (ie $\left.\xi=\xi_{i}\right)$. \\ $X\left(\theta_{i}, \xi\right)=X_{i} \quad$ Random process reduced to a simple random variable once the variable $\theta$ is fixed \\ $\tilde{X}\left(f_{\theta}\right)$ \\ $\mathscr{D}_{X X}\left(f_{\theta}\right)$ \\ $\left\lceil x^{*}\right.$ \\ Discrete Fourier Transform of realisation $X_{\xi}(\theta)$ normalized by the length of the realisation \\ Power spectral density of random signal $X$ at cyclic frequency $f_{\theta}$ \\ Rounding to the highest integer value \\ Complex conjugate
}

\section{Introduction}

In the last twenty years, Instantaneous Angular Speed (IAS) has been prospected for different purposes and by different means. The spectral observation of the IAS signal has been revealed as an effective tool for engine combustion related diagnosis [1] and recently appeared as a promising tool for bearing heath monitoring, the running conditions being stationary or not [2]. Yuhua et al. reviewed angular speed measurement methods by categorizing them into two broad groups: timer/counter-based methods and ADC-based methods. It will be shown in this paper that this categorization could rather be made between the system counting the number of pulses in a given time duration and those measuring the Elapsed Time for a single cycle of encoder signal [3]. This simplification therefore distinguishes the speed measurement systems sampling data in the time domain from those sampling data in the angular domain. The latter is the only concerned with the results obtained in the present paper. Angular signals are especially suited to deal with non stationary rotating mechanism and IAS based monitoring is even more attracting since it is remarkably easy to obtain through these techniques [4].

As far as the authors know, there is no way to analogically filter an angular domain IAS signal; this makes it prone to aliasing, a phenomenon that has not been detailed yet regarding this technique, the only proposed answer being to increase the angular sensor resolution to the highest possible value [5]. This solution may yield a counter productive effect over the signal spectral observation since other sources of perturbation could depend on the sensor resolution in a negative way. In this regard, Youssef et al. brought a visual description of the spectral noise of the IAS obtained through an angular domain observation, distinguishing the discrete error coming from the angular sensor irregularities and the large band perturbation induced by the derivation phenomenon [6]. Nevertheless, the relation between the system parameters and the signal-to-noise ratio has not been studied and this ignorance appears as a major drawback for this attractive technique, especially since the observed IAS amplitude of variation have appeared alarmingly weak.

This paper proposes an other step towards the determination of the overall precision of the IAS obtained through the Elapsed Time technique. Once the measurement methods have been described, the existence of aliasing and its influence is theoretically presented. This first step aims at understanding the relation between the actual IAS variations and those, possibly aliased, observed on its spectral observation. Subsequently, the quantization phenomenon is tackled: the case of stationary speed is shown to lead to inevitable perturbations which take the shape of spectral ghost components. The theory of non stationary speed condition is eventually addressed with a statistical approach. The 
results are finally validated numerically and experimentally on an automotive gear-box test rig. Amongst the practical and beneficial results, the speed variations experienced by the mechanical system along with the quantization process will now be understood as valuable for the monitoring process.

\section{Instantaneous Angular Speed monitoring system}

\subsection{Angular speed sensor}

IAS can be obtained from several methods. All of them are based on the observation of a rotating system through a sensor able to convert the angular position into an electrical signal. Most of these sensors are optical encoders, magnetic pick-ups and hall effect tooth wheels [7]. If optical sensors are known to reach higher resolution and precision, the other two can often be set in more extreme conditions. Typically, optical encoders are of common use to precisely synchronise asynchronous generator converter with the rotor speed while phonic wheels are used to warn the operator in the extreme case of a mechanical coupling breakdown. Other measurements are done with costly laser vibrometers [8], low resolution resistive potentiometric sensor [9] or resolver whose precision and magnetic sensibility decrease as soon as the resolution increase [10].

The terminology angular sensor is of use in this article to associate every elements able to convert an angular position into an electrical signal with the results presented hereafter. The signal conditioning applied on these angular sensor includes several common steps, resumed in Fig. 1, possibly ameliorated by an interpolation process to yield a square signal whose resolution is higher than the physical one, as mentioned in [11].

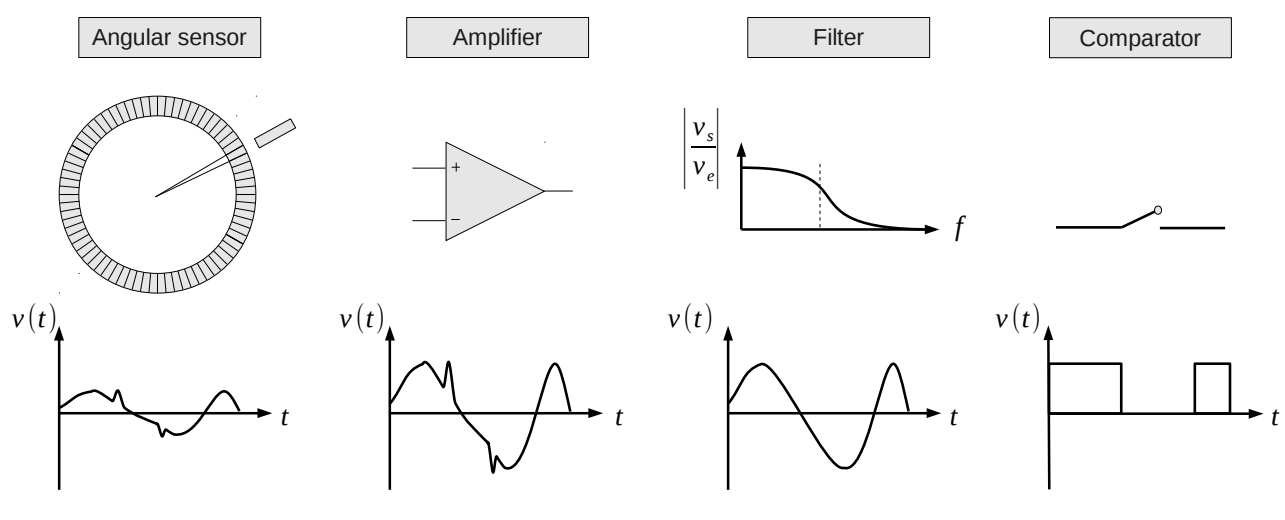

Figure 1: Conditioning process of an angular sensor.

Although the result quality may depend on the selected sensor type, it is important to notice that this technological choice is almost independent of the method chosen to compute the instantaneous angular speed.

\subsection{IAS Computation methods}

According to the acquisition system available to acquire the electrical signal coming from the angular sensor, several methods have been developed to estimate the IAS. In [3], Li et al. give an exhaustive list of these methods that could be sorted in another relevant manner, aiming at distinguishing those able to deal with non stationary conditions.

\subsubsection{Time dependant methods}

This group of methods represents all those aiming at sampling the speed signal in the time domain. The intuitive frequency meter technique measures the angle displacement of the monitored shaft during a steady period of time counting the number of angular signal pulses obtained from the angular sensor. More efficiently, the IAS can be estimated along with the instantaneous frequency of the time sampled angular sensor signal. This method relies on the use of tools dedicated to the analysis of frequency modulated signal, such as the phase and amplitude envelope demodulation [12]. Although this method is easy to implement, it is limited by the following drawbacks: 
- the carrier frequency must be stable enough not to out-range the bandwidth of the filter used to demodulate the angular sensor signal. This method is dedicated to mechanisms whose speed trend is stationary.

- The filter used to extract the carrier frequency must be correctly chosen and parametrized. Although the Gaussian window has been shown to yield the best results for the peculiar application described in [13], its width is still difficult to tune since it has a direct influence over the signal to noise ratio.

- If IAS is used to monitor cyclic components running at varying speed conditions, the signal must be re-sampled in the angular domain before the mechanical elements can be monitored along their respective characteristic frequencies.

The time dependant methods present clear weaknesses when applied on non stationary mechanisms, although the authors are aware that their relevance is not to be proven in the more common case of steady speed machinery. This section introduces the need to work with the Elapsed Time method, since it is intrinsically carried in the angle domain.

\subsubsection{Angle dependant methods}

On the opposite, the angle dependant methods stand for all those aiming at sampling the speed signal in the angle domain. From a general point of view, angular speed in the angular domain can be expressed as follows:

$$
\omega(\theta)=\frac{d \theta}{d t}(\theta)
$$

This is practically achieved through the measurement of the Elapsed Time $\tau(\theta)$ between two consecutive rising edges issued from the angular sensor describing the angle $\Delta \theta$.

$$
\bar{\omega}(\theta)=\frac{\Delta \theta}{\tau(\theta)}
$$

In a first embodiment, illustrated in Fig. 2 the rising edges can be located on a classical ADC acquisition board, through the use of zero crossing techniques. This method is peculiarly adapted to computing IAS from angular sensor delivering sinus shape signals [14]. In a second embodiment, illustrated in Fig. 3, where the encoder signal feeds a digital acquisition board and directly manages a high frequency counter, the signal can be processed by counting rates from megahertz to gigahertz. This method will be of use in the rest of the paper.

(a)

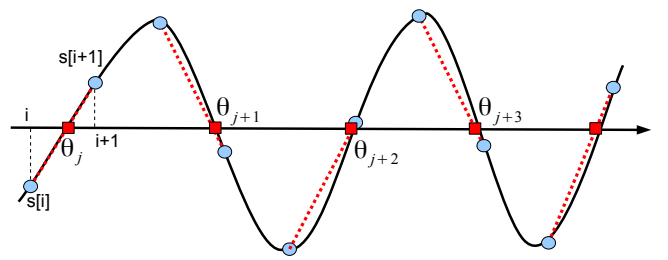

(b)

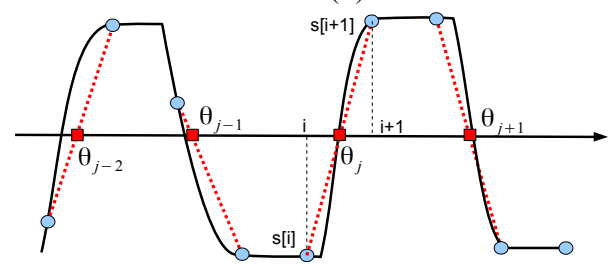

Figure 2: Schematic example of signal edges detection using an ADC acquisition board applyed on: (a) sinus shape signal, (b) square signal.

Whether the angular sensor is considered to deliver a digital or an analogical signal, the rotating speed is always estimated for each rising edge of the encoder signal with respect to Eq. (3),

$$
\bar{\omega}_{i}=\frac{2 \pi}{R \cdot \tau_{i}}, \quad \hat{\omega}_{i}=\frac{2 \pi}{R \cdot \hat{\tau}_{i}}
$$

where $R$ is the resolution of the encoder and $\tau_{i}$ is the time difference between the $i^{\text {th }}$ and the $(i-1)^{\text {th }}$ segment delivered by the tachometer which, disregarding geometrical errors in the tachometer gratings, exactly correlates with the IAS of the shaft carrying the sensor. 


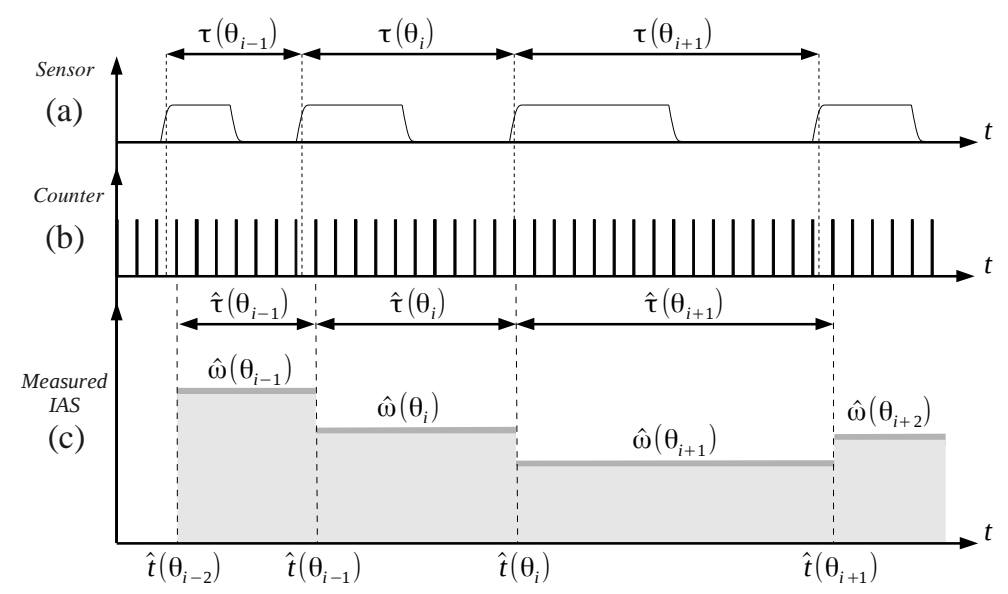

Figure 3: Instantaneous Angular Speed measurement with the Elapsed Time method. (a) square voltage from the tachometer. (b) High frequency clock pulses. (c) IAS estimation.

Besides, it must be understood that all the traditional habits linked to classical work in the spectral domain must be revised since the signal is not sampled in the time domain but in the angular one. The angular sampling nature of the measurement allows the small speed variations induced by a fault to be steadily correlated to the IAS measurement, the global speed of the mechanism being stationary or not. The IAS Fourier transform is the distribution of the IAS variations along the cyclic frequency axis. The sampling cyclic frequency is ruled by the encoder resolution rather than the counting/sampling frequency of the acquisition board.

Then, regarding Eq. (3) and Fig. 2, two specificities of the Elapsed Time measurement can be emphasized: the measured IAS is an averaged one according to time difference on angular increments, and the time difference is a discrete value limited by counter clock frequency. Next sections detail how the IAS spectrum is affected by this two specificities, especially the filtering effect due to average and the quantization effect due to counter clock frequency.

\section{Filtering effect due to Elapsed Time measurement}

\subsection{Filtering effect}

According to Eq. (2), the measured IAS is an averaged value of the actual IAS, i.e. the latter will be low-pass filtered by the measurement. The aim of this part is to demonstrate exactly the shape of this filtering effect. The hypothesis is made that IAS is quite constant, so as to develop first order approximations. Equations related to this hypothesis are:

$$
\omega(\theta)=\omega_{0}+\delta \omega(\theta),
$$

with:

$$
\left|\frac{\delta \omega(\theta)}{\omega_{0}}\right|<<1 \quad \forall \theta .
$$

Considering this hypothesis, the first-order approximated spectrum of the estimated IAS can be simply expressed as the following equation, as demonstrate in Appendix A:

$$
\bar{\Omega}\left(f_{\theta}\right)=\Omega\left(f_{\theta}\right) \cdot e^{-j \pi f_{\theta} \Delta \theta} \cdot \operatorname{sinc}_{\pi}\left(f_{\theta} \Delta \theta\right) .
$$

As a conclusion, it can be observed that, for an angular speed with small variations, the Elapsed Time technique creates a low-pass filter with a $\operatorname{sinc} \pi$ shape based on $\Delta \theta$ resolution of the encoder. 


\subsection{Aliasing consideration and numerical validations}

To the authors knowledge, there is yet no solution to analogically filter the IAS signal before the sampling process. Previous part shows that the Elapsed Time technique provides low-pass filter effect, which can be used to correctly design the experimental set-up and limit aliasing.

Considering the case of an angular sampled IAS obtained with Elapsed Time technique, the spectrum of the measured IAS corresponds to Eq. (7). It is noted that angular sensor resolution $\Delta \theta$ drives both filter and aliasing.

$$
\hat{\Omega}\left(f_{\theta}\right)=\Omega\left(f_{\theta}\right) \cdot e^{-j \pi f_{\theta} \Delta \theta} \cdot \operatorname{sinc}_{\pi}\left(f_{\theta} \Delta \theta\right) * \delta_{\frac{1}{\Delta \theta}}\left(f_{\theta}\right) .
$$

To illustrate Elapsed Time technique effect under angular sampling conditions, numerical simulations of IAS are defined as follows:

$$
\omega=\omega_{0}+0.5 \cdot \sum_{k=1}^{2 . R / 9} \sin (3 k \theta),
$$

with $R=256 \mathrm{pulses} / \mathrm{rev}$, the encoder resolution, and $\omega_{0}$ that can be set to $200,100,50$.

The numerical IAS and the corresponding (simulated) measured signal for $\omega_{0}=200$ can be observed in the angular domain in Fig. 4. It is noted that the IAS variation amplitude is of about $60(|\delta \omega<30|)$. The small variation assumption is variously verified for the IAS as $\left|\delta \omega / \omega_{0}\right|$ varies from $15 \%$ to $55 \%$.

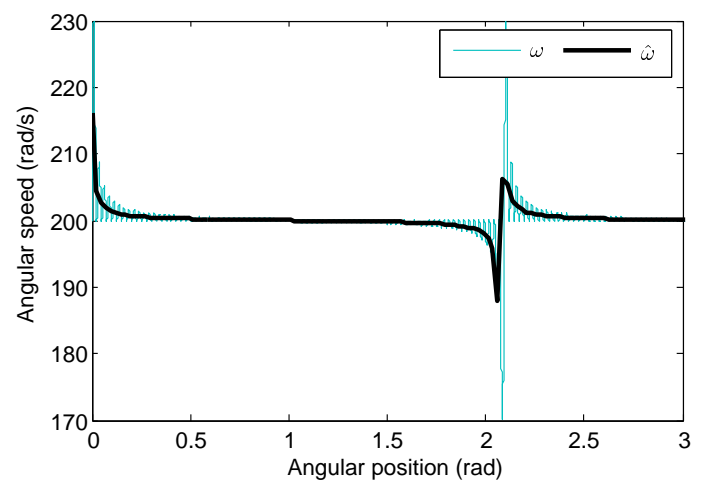

Figure 4: Effect of measurement of angular speed on signal spectrum.

Spectra of the simulated true and measured IAS are presented in Fig. 5. The cardinal sine shape attenuation is observed for all the measured signals. When the mean value of angular speed is of the same order of magnitude as the angular speed variation, spectral values are a bit higher than expected, but the cardinal shape is globally respected.

Aliasing is also clearly present, as a second cardinal sine envelope centred on sampling frequency is observed. Consequently, the spectrum of the measured IAS may provide a good picture of the true IAS spectrum at low frequency, but it appears that peaks near Nyquist frequency will be of the same amplitude, whatever they are from direct part or aliasing part.

If the frequency content of the IAS is neither known nor expected, the best approach to analyse IAS spectrum is to consider the first quarter spectrum (cyclic frequencies lower than $1 /(4 . \Delta \theta)$ ), for which aliased peak amplitudes are $14 \mathrm{~dB}$ less compared to direct part of the spectrum. Resolution of the angular encoder can also be choose as four times the higher frequency expected.

However, when the resolution of the sensor cannot be increased to such a level, it is now possible to rectify the observed IAS variation amplitude corresponding to any cyclic phenomenon as soon as the original cyclic frequency (i.e. before aliasing) is known. This might be of extreme help with the use of low resolution encoder such as classical tooth wheel inductive sensor. 


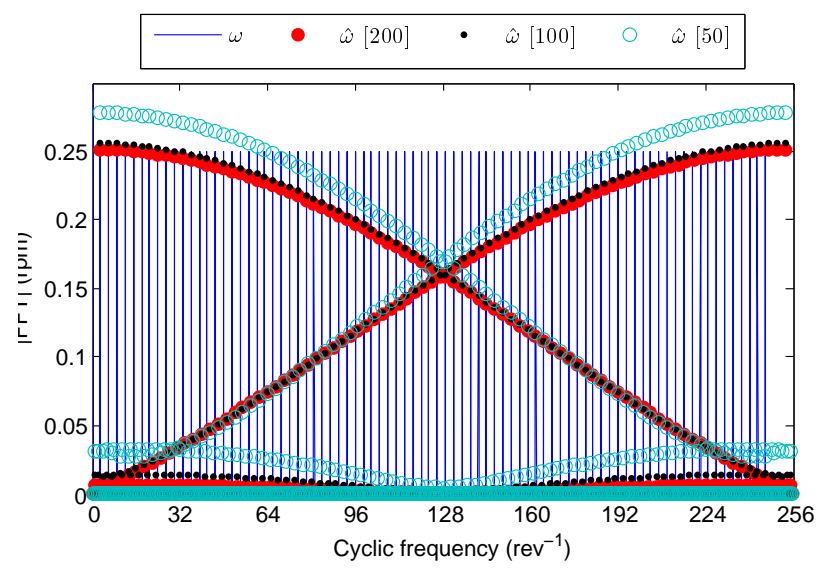

Figure 5: Numerical validation of cardinal sine shape attenuation in amplitude spectrum for different mean speed values.

\subsection{Experimental considerations}

\subsubsection{Presentation of the test rig}

The test bench dedicated to this study can be observed in Fig. 6. It is made up of an automotive gearbox, driven by an electrical motor mounted in an open loop design allowing generation of an acyclic excitation as well as a speed ramp. The gearbox is loaded by means of an electrical generator which is torque monitored. Most of torsional vibrations induced by the generator are mechanically filtered with a torsion rod surrounded by two inertia wheels. Revolution speed of the whole kinematic chain can be controlled over a large range of operating conditions. The gearbox presents two reduction stage. The first reduction stage with four different gear ratios is set to a $27 / 31$ gear ratio, using the $3^{\text {rd }}$ gear. The second stage is fixed and loaded with a $15 / 64$ gear ratio.

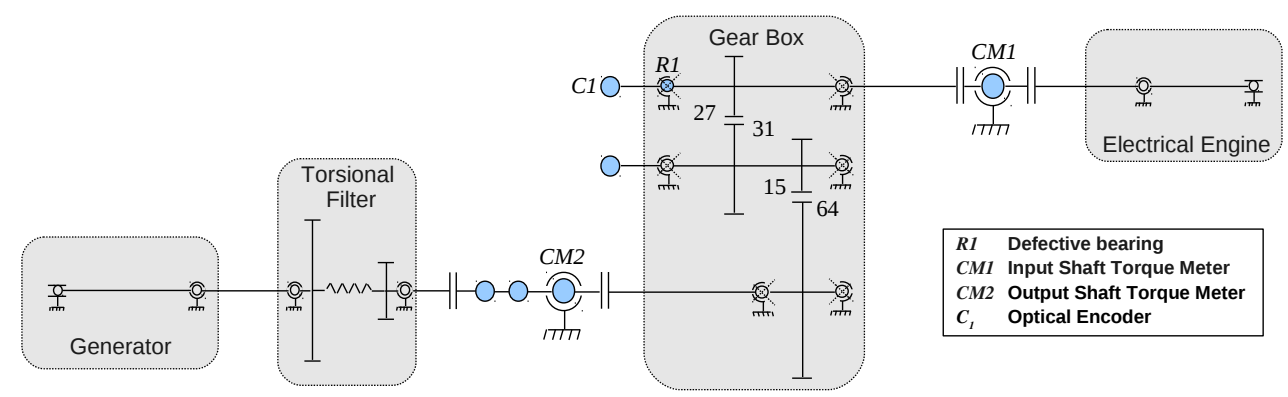

Figure 6: Kinematic scheme of the automotive test rig.

The gearbox is equipped with different transducers on the three shafts as shown in Fig. 6 . Two torquemeters/tachometers are located around the gearbox to serve the command feedback loop. One optical encoder offering through shaft design is mounted on the transmission input shaft and delivers 2048 pulses per revolution through a regular TTL signal. The FPGA based acquisition system can read the digital input with a rate up to $120 \mathrm{MHz}$ and can artificially reduce the resolution of the encoder.

The typical experimental test is a run up from 200 to $1000 \mathrm{rpm}$, as shown in Fig. 7. For the validation of filter effect and aliasing results, the signal with the best precision is used: resolution of $2048 \mathrm{ppr}$ and counting frequency of $120 \mathrm{MHz}$. Based on this latter, low resolution encoders are simulated to observe the effect of aliasing on gear mesh frequency peaks, with the aim to restore the correct amplitude of the monitored peaks. Signals corresponding to low resolution encoder are obtained by cumulating many consecutive $\tau_{i}$. 


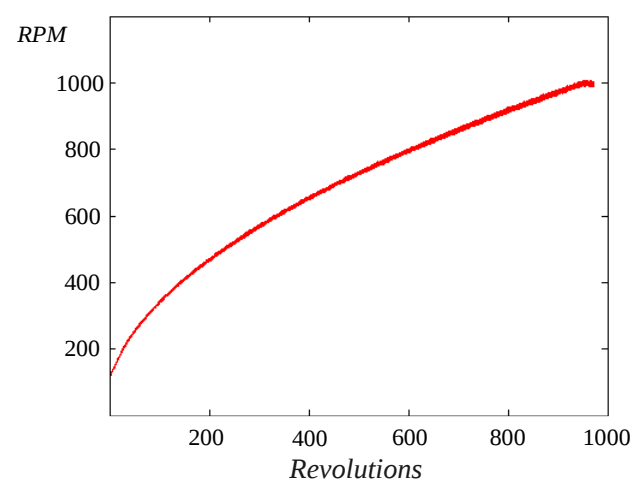

Figure 7: Running speed conditions).

\subsubsection{Experimental validations of Elapsed Time filter effect under angular sampling}

The spectrum of the full resolution signal $\left(\mathrm{R}=2048 \mathrm{ppr}, F_{c}=120 \mathrm{MHz}\right)$ can be observed in Fig. 8 . It is considered as the true one in the observed zone, since values of the direct cardinal sine are about one, values of the aliased cardinal sine are very low and the signal may provide no harmonic at very high frequencies (2000 events per revolution). The two first main peaks at $13.065 \mathrm{rev}^{-1}$ and $27 \mathrm{rev}^{-1}$ correspond to gear mesh frequencies of second stage (15 teeth gear) and first stage (27 teeth gear) respectively, observed through $C 1$ optical encoder. These peaks will be tracked through filtering and aliasing effect, with the reduction of encoder resolution.

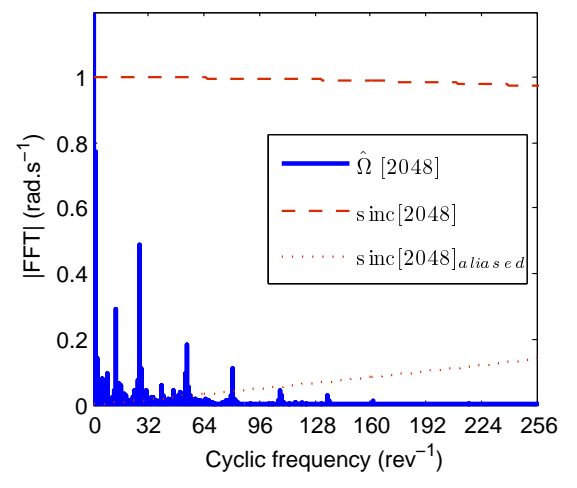

Figure 8: Spectral contents of the experimental test signal.

Amplitude spectrum of the measurement simulated with 8 points per revolution is plotted in Fig. 9, in comparison with true spectrum. In that case, gear mesh frequencies occur at $2.935 \mathrm{rev}^{-1}$ (second stage) and $3 \mathrm{rev}^{-1}$ (first stage), and their amplitudes become very small. Theoretical formula (cardinal sine shape, Eq. (7)) is used to find the original amplitude for different simulated resolution. Results can be analysed in table 2.3.2.

It comes that for the second stage, the amplitude of gear mesh frequency is corrected with a very high precision (error lower than 2\%). Nevertheless for the first stage, the error in the corrected values raises over $40 \%$ as soon as the peak aliased. Indeed, as the encoder is mounted on the 27 teeth gear shaft, the peak will always aliased on a harmonic of the rotation, whose amplitude is not negligible: $\hat{\Omega}(5)$ and $\hat{\Omega}(3)$ respectively are quite equivalent whatever a mesh frequency aliased $(\mathrm{R}=32$ and $\mathrm{R}=8$ respectively) or not $(\mathrm{R}=2048)$. In the particular case of the Fig. 9 , it can be seen that a peak exists at cyclic frequency $3 \mathrm{rev}^{-1}$, that will mix with the aliased peak of 27. Moreover, the peak at cyclic frequency $5 \mathrm{rev}^{-1}$ will also aliased on cyclic frequency $3 \mathrm{rev}^{-1}$. It can be conclude that the estimation of true amplitude of peculiar harmonic with low resolution encoder is possible only if it aliases on a free cyclic frequency. Mesh frequencies that don't concern encoder shaft and bearing frequencies are part of these peaks that can 


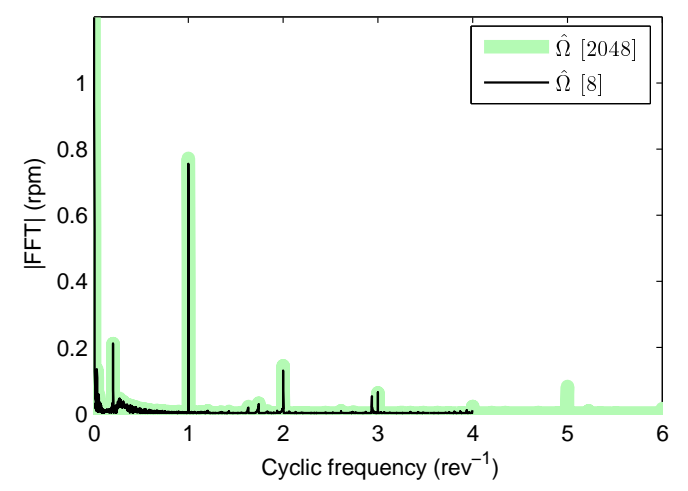

Figure 9: Experimental validation of aliased shape spectrum.

be estimated.

\begin{tabular}{ccccccc}
\hline Resolution & 2048 & 128 & 64 & 32 & 16 & 8 \\
\hline \hline sinc[13.065;R] & 1 & 0.983 & 0.933 & 0.748 & 0.213 & 0.178 \\
$\hat{\Omega}(13.065)$ & 0.292 & 0.287 & 0.272 & 0.219 & - & - \\
$\hat{\Omega}(2.935)$ & 0 & 0 & 0 & 0 & 0.0621 & 0.0512 \\
$\Omega_{\text {est. }}(13.065)$ & 0.292 & 0.292 & 0.292 & 0.293 & 0.292 & 0.288 \\
error & 0 & 0 & 0 & $0.3 \%$ & 0 & $1.4 \%$ \\
\hline sinc[27;R] & 1 & 0.928 & 0.732 & 0.178 & 0.157 & 0.087 \\
$\hat{\Omega}(27)$ & 0.491 & 0.457 & 0.355 & - & - & - \\
$\hat{\Omega}(5)$ & 0.081 & 0.081 & 0.080 & 0.123 & 0.117 & - \\
$\hat{\Omega}(3)$ & 0.061 & 0.061 & 0.061 & 0.066 & 0.060 & 0.0629 \\
$\Omega_{\text {est. }}(27)$ & 0.491 & 0.492 & 0.485 & 0.691 & 0.745 & 0.723 \\
error & 0 & $0.2 \%$ & $1.2 \%$ & $\mathbf{4 0 \%}$ & $\mathbf{5 2 \%}$ & $\mathbf{4 7 \%}$ \\
\hline
\end{tabular}

Table 1: Estimation of the actual amplitude of gear mesh frequency peaks with low resolution encoder. Cardinal sine are used to find the original value before attenuation. If the signal aliased, value is read at the aliased position.

To resume, the Elapsed Time measurement works as a low-pass filter based on $\operatorname{sinc}_{\pi}$ shape and encoder resolution. Thus, a correction is possible to restore the correct amplitude of peculiar peaks if spectral content is known. If the tracked peak aliased, it mustn't superpose with a low frequency peak if the correction is expected. Low resolution encoder can thus be used for monitoring bearing and gears that doesn't belong to shaft encoder (non-integer ratio from the encoder point of view).

These results about Elapsed Time technique concern the global behaviour of the measurement, thanks to time lap estimation between two successive angular positions of the encoder. This time increment was considered as the exact value, but it is experimentally estimated through countering high frequency clock. The aim of next part is to quantify the effect of the precision of time increment estimation on the measured signal.

\section{Quantization precision}

The estimation of the time separating two consecutive rising edges is rounded accordingly to the counting frequency and introduces a quantization error. In other words, this phenomenon is induced by the error made during the measurement of an instant through the use of a counter. It is very unlikely that the impulses delivered by the counter are perfectly synchronised with those delivered by the angular sensor. Enlightened in Fig. $3, \hat{\tau}\left(\theta_{i}\right)-\tau\left(\theta_{i}\right)$ gathers the delays taken by the counter to observe the rising edge of two consecutive angular sensor impulses. This difference is what the authors call the quantization error. 
The instantaneous angular speed monitoring is based on the spectral observation of the speed signal. The aim of this part is therefore to understand the influence of this phenomenon over the speed signal once it has been transformed in the spectral domain. In a first approach, the IAS is considered constant. Although the chance to deal with such conditions are very low in reality, this case aims at introducing the statistical approach which is used in a second step.

\subsection{Stationary system}

In order to dress properties on the quantization phenomenon when the IAS is constant, two different cases are to be distinguished:

- an integer number of clock tops exactly separates two consecutive segment of the encoder,

- a rational and non integer number of clock tops exactly separates two consecutive segments.

The third case where the number of clock ticks separating two consecutive segments is neither integer nor rational is not considered. This last option does not seem of any interest since the two first cases are already very unlikely to happen in real world.

\subsubsection{Integer number of clock tops}

By assumption that a whole number of clock tops exactly separates two consecutive encoder segments, it comes:

$$
\tau=p \cdot T_{c},
$$

where $p$ is a positive integer. Thus, the real time between the $i^{t h}$ rising edge and the beginning of the acquisition start depends on $t_{0}=t\left(\theta_{0}\right)$, the time between the first rising edge and the counter start. There exist $p \in \mathbb{N}$ and $t_{0}$ an integer between 0 and $p \cdot T c$ such as:

$$
\forall i \in[|1, n|], t_{i}=i \cdot p \cdot T_{c}+t_{0} .
$$

The quantization phenomenon is introduced with the use of the ceiling function $\lceil x\rceil$, which rounds $x$ to the next integer. The time separating the $i^{\text {th }}$ rising edge and the counter start is equal to:

$$
\hat{t}_{i}=\left\lceil\frac{i \cdot p T_{c}+t_{0}}{T_{c}}\right\rceil T_{c}=i \cdot p \cdot T_{c}+\left\lceil\frac{t_{0}}{T_{c}}\right\rceil \cdot T_{c} .
$$

As illustrated in Fig. 3, the measured time $\hat{\tau}_{i}$ corresponding to every segment $\theta_{i}$ is obtained from finite difference of the consecutive rising time $\hat{t}_{i-1}$ and $\hat{t}_{i}$. From the previous equations, it finally comes:

$$
\hat{\tau}_{i}=p \cdot T_{c}=\tau_{i}
$$

The equality $\hat{\tau}_{i}=\tau_{i}$ is then verified and will be preserved by the Fourier Transform. In this very unlikely case, the spectral observation of the IAS is not affected by the quantization approximation.

\subsubsection{Rational number of clock tops}

Assuming that a rational and non integer number of clock ticks exactly separates two consecutive segments, it comes:

$$
\exists p, q \in \mathbb{N}^{2} / \tau_{i}=\frac{p}{q} \cdot T_{c},
$$

with $p$ and $q$ coprimes. In this case, the quantization perturbation is shown in Appendix B.1 to be non zero and periodic of period $q$. The quantization phenomenon is therefore enlightened on the spectral observation of the IAS through, at least, the set of harmonic whose fundamental frequency equals $R / q$ events per revolutions. It is clear that this proof does not mean this angle-periodic perturbation is the only one induced by the quantization phenomenon. One of them is enhanced, maybe the easiest to point out, and indicates that the quantization process induces perturbations that might be wrongly interpreted as mechanical cyclic phenomena on the amplitude spectrum of the IAS. These properties are numerically shown for a better understanding. 


\subsubsection{Numerical validation}

Two signals are simulated accordingly to the parameters presented in Tab. 2. Although the signals present very close angular speed, the first simulation meets the criteria detailed in part 3.1.1 and the second meets those presented in part 3.1.2. The sequence $\tau_{i}$ and $\hat{\tau}_{i}$ of both simulations are respectively displayed in Fig. 10 . In the first case, the relation $\tau_{i}=\hat{\tau}_{i}$ is verified and the spectral observation of the IAS does not tell more than the average rotating speed. In the second case, the periodicity of the sequence is evidenced as a sawtooth wave. The amplitude of this wave is equal to the quantization spacing: $T_{c}$. The curve labeled $t_{i}-\hat{t}_{i-1}$ represents the time separating the $i^{t h}$ rising edge and the previous measured rising edge. Taking the form of a sawtooth curve, the resetting of the accumulated quantization error is made once the time constant of the counter is reached.
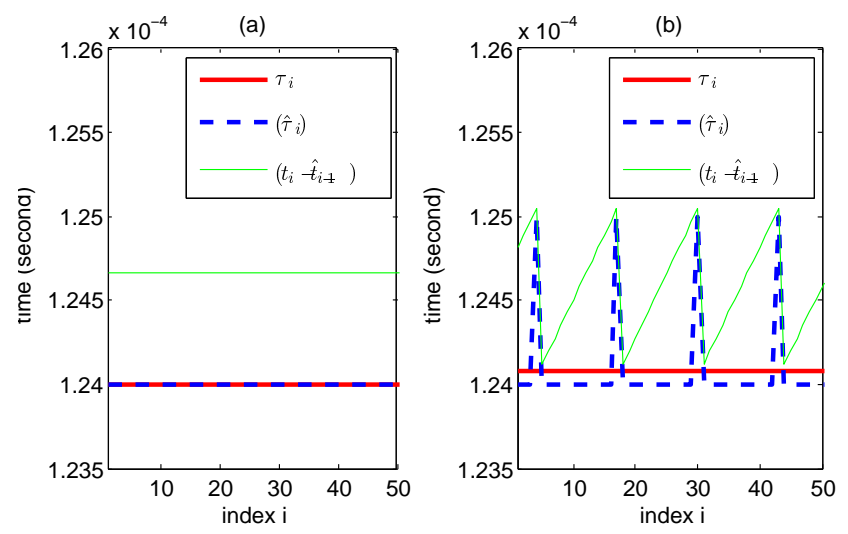

Figure 10: Simulations with (a) an integer number of counter pulses, (b) a rational number of pulses along each angular sensor period.

\begin{tabular}{|c|c|c|}
\hline Parameters & Signal 1 & Signal 2 \\
\hline Counter frequency $\left(F_{c}\right.$ in $\left.\mathrm{Hz}\right)$ & $1 \cdot 10^{6}$ & $1 \cdot 10^{6}$ \\
\hline Angular sensor Resolution ( $R$ in Pulse.rev ${ }^{-1}$ ) & 100 & 100 \\
\hline Signal length $(N)$ & 1000 & 1000 \\
\hline angular speed ( $\omega$ in $\left.\mathrm{rad} . \mathrm{s}^{-1}\right)$ & 80.6 & 80.65 \\
\hline Numerator $(p)$ & 124 & 1613 \\
\hline Denominator $(q)$ & 1 & 13 \\
\hline
\end{tabular}

Table 2: Parameters used for the numerical simulation.

The amplitude spectrum corresponding to the second simulation is presented in Fig. 11 where the spectral components multiple of $R / q$ are evidently visible. Although this result is unambiguously validated with simulated measurements, experimental testing will hardly present such a steady operating speed. This comb perturbation is more likely to threaten simulations obtained from a mechanical system model.

Without mentioning the unlikely case of a speed exactly synchronised with the counter frequency, the quantization phenomena creates non zero angle-periodic artefacts even on a steady speed IAS signals. It is possible to localize the cyclic frequency of some of these artefacts using the denominator of the ratio between the angular speed and the counting frequency. Unfortunately, the slightest angular speed deviation affects this ratio $R / q$ in an unforeseeable manner which will be qualified as random by the authors. Hence, the angular speed variations of the trend as it is seen in Fig. 7 are suspected to introduce a random component in the cyclic frequency localization of the artefacts induced by the quantization.

\subsection{Non stationnary case}

A statistical approach is undertaken to manipulate the random perturbation of the quantization, in case of non stationary behaviour. Practically, this approach is expected to estimate a threshold distinguishing the spectral compo- 


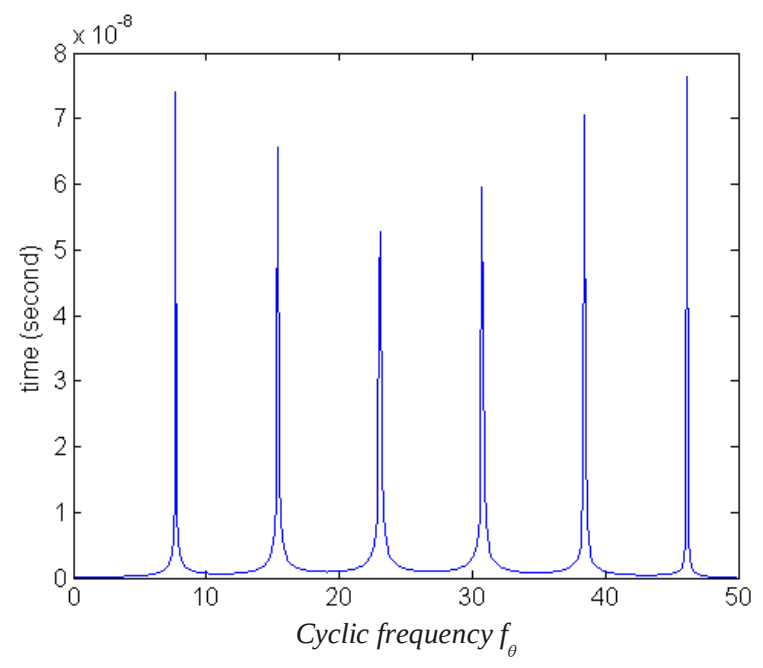

Figure 11: Amplitude spectrum of the simulation presenting a rational number of impulses along each angular sensor period $\left(R / q=7.7 \mathrm{rev}^{-1}\right)$.

nents induced by the quantization perturbation from the real mechanical information. The tools used to reach this aim are detailed in a first section

\subsubsection{Statistics basics}

In probability theory, the central limit theorem states that the average of a sufficiently large number of independent random variables, each with finite mean and variance, will be approximately normally distributed. Let $X_{1}, X_{2}, \ldots, X_{n}$ be a sequence of $n$ independent and identically distributed random variables each having finite valued expectation $\mu$ and variance $\sigma$. The sum of the $n$ random variables converges in distribution to the standard normal distribution of expectation $n \mu$ and standard deviation $\sigma \sqrt{n}$. This theorem is irrespective of the shape of the common distribution of the individual terms of the sequence.

Moreover, the chi-square distribution with $\mathrm{k}$ degrees of freedom is the distribution of a sum of the squares of $\mathrm{k}$ independent standard normal random variables. Let $X_{1}, X_{2}, \ldots, X_{n}$ be a sequence of $n$ independent, standard normal random variables. Then, the sum of their squares is distributed according to the chi-square distribution with $\mathrm{k}$ degrees of freedom.

\subsubsection{Quantization error in a simple form}

In a first step, the simpler case of a unique random variable is considered. This corresponds to the quantization error $\varepsilon_{i}$ induced during the estimation of the variable $t_{i}$ :

$$
\varepsilon_{i}=t_{i}-\bar{t}_{i}=t_{i}-\left\lceil\frac{t_{i}}{T_{c}}\right\rceil \cdot T_{c} .
$$

The random variable $\varepsilon_{i}$ is assumed to follow an uniform law on the interval $\left[0, T_{c}\right]$. Commonly proposed in quantization theory, this assumption is especially realistic if the quantization spacing $T_{c}$ is small in front of the dynamic of the signal and if the signal stands relatively uniformly in this dynamic. Hence, this assumption is strong and it has to be enlightened that a steady speed measurement is intrinsically opposed to this criterion: results obtained from this hypothesis are expected to differ from those obtained in part 3.1 .

The previous hypothesis let the authors assume the random signal $\varepsilon$ is stationary and ergodic, it can therefore be characterised by means of the successive moments of the random variable $\varepsilon_{i}$. Similarly, this random signal is characterised in the spectral domain through the use of its PSD $\mathscr{D}_{\varepsilon \varepsilon}\left(f_{\theta}\right)$. This will be enough to define the quantization threshold $v_{\varepsilon \varepsilon}\left(f_{\theta}\right)$ that might eventually be compared to the PSD $\mathscr{D}_{\tau \tau}\left(f_{\theta}\right)$ of the signal representative of the mechanism speed variations. From the periodogram of a realisation $\varepsilon_{i}(\theta)$, the random signal PSD is estimated such as:

$$
\mathscr{D}_{\varepsilon \varepsilon}\left(f_{\theta}\right) \triangleq N\left(\tilde{E}\left(f_{\theta}\right) \cdot \tilde{E}^{*}\left(f_{\theta}\right)\right) .
$$


The complex spectral coefficients $\tilde{E}\left(f_{\theta}\right)$ are obtained after summation of every terms of the random process $\varepsilon_{i}(\theta)$ respectively weighted by a complex exponential. Let $a\left(f_{\theta}\right)$ and $b\left(f_{\theta}\right)$ be the independent random processes standing for the real part and the imaginary part of the process $E\left(f_{\theta}\right)$. The central limit theorem states that $a\left(f_{\theta}\right)$ and $b\left(f_{\theta}\right)$ converge in distribution to the standard normal distribution of expectation 0 and variance $\sigma^{2} / 2 N=T_{c}^{2} / 24 N$. The PSD $\mathscr{D}_{\varepsilon \varepsilon}\left(f_{\theta}\right)$ is estimated from the random process realisation $\varepsilon_{i}(\theta)$ by the reduced relation:

$$
\mathscr{D}_{\varepsilon \varepsilon}\left(f_{\theta}\right) \triangleq \frac{\sigma^{2}}{2} \cdot\left(\left(a\left(f_{\theta}\right) \cdot \frac{\sqrt{2 N}}{\sigma}\right)^{2}+\left(b\left(f_{\theta}\right) \cdot \frac{\sqrt{2 N}}{\sigma}\right)^{2}\right) .
$$

Let $X\left(f_{\theta}\right)$ be the sum of two squared variables, respectively following two standardised Gaussian laws, and defined such as:

$$
X\left(f_{\theta}\right)=\left(a\left(f_{\theta}\right) \cdot \frac{\sqrt{2 N}}{\sigma}\right)^{2}+\left(b\left(f_{\theta}\right) \cdot \frac{\sqrt{2 N}}{\sigma}\right)^{2} .
$$

The chi-square theorem states that $X\left(f_{\theta}\right)$ is distributed according to the chi-square distribution with 2 degrees of freedom. Therefore, the probability density of $X\left(f_{\theta}\right), f_{X}=1 / 2 \cdot \exp (-t / 2)$ can be used to determine the threshold $\eta_{X}$ of the random variable $X(\theta)$ able to distinguish quantization noise from real information, under the probability $\alpha$ :

$$
\left.\begin{array}{l}
P\left(X<\eta_{X}\right)=1-\alpha \\
P\left(X<\eta_{X}\right)=\int_{0}^{\eta_{X}} f_{\chi^{2}(2)}(t) \mathrm{d} t
\end{array}\right\} \Longrightarrow \eta_{X}=-2 \ln \alpha .
$$

From Eq. (16) and (18), the threshold $\eta_{\mathscr{D} \varepsilon \varepsilon}$ is defined to test the hypothesis that a spectral component can be induced by the quantization error under the probability $\alpha$ :

$$
\eta_{\mathscr{D}_{\varepsilon \varepsilon}}=-\sigma^{2} \cdot \ln (\alpha)
$$

In practical terms, the PSD $\mathscr{D}_{\varepsilon \varepsilon}\left(f_{\theta}\right)$ has a ratio of spectral components exceeding the threshold $\eta_{\mathscr{D}_{\varepsilon \varepsilon}}$ close to $\alpha$. The influence of the quantization over the random process $\varepsilon_{i}(\theta)$ is similar to the one over the real measurement $t_{i}(\theta)$. The following paragraph aims at describing the influence of quantization over the spectral observation of the measurement signal $\tau_{i}(\theta)$.

\subsubsection{Quantization error in a finite difference form}

In the previous section the quantization noise has been described when applied on the variable $t_{i}$. Nevertheless, the IAS monitoring is performed on the time difference $\tau_{i}$, another random variable defined by the finite difference. $t_{i}$ and $t_{i-1}$ are considered independent variables, and once again, this assumption can not be applied to the stationary case presented in section 3.1. The results presented in Eq. (19) will be used to determine the same kind of distinction criterion for the random signal $\Delta \varepsilon$, defined by its random variable:

$$
\left.\begin{array}{rl}
\Delta \varepsilon_{i} & =\tau_{i}-\hat{\tau}_{i} \\
\hat{\tau}_{i} & =\hat{t}_{i}-\hat{t}_{i-1} \\
\tau_{i} & =t_{i}-t_{i-1}
\end{array}\right\} \Longrightarrow \Delta \varepsilon_{i}=\varepsilon_{i}-\varepsilon_{i-1} .
$$

The demonstration, available in Appendix B.2, draws a relation between the PSD of $\Delta \varepsilon$ and the PSD of $\varepsilon$ :

$$
\mathscr{D}_{\Delta \varepsilon \Delta \varepsilon}\left(f_{\theta}\right)=4 \cdot \mathscr{D}_{\varepsilon \varepsilon}\left(f_{\theta}\right) \cdot \sin \left(\pi f_{\theta} \Delta \theta\right)^{2}
$$

From Eqs. (19) and (21), it is possible to estimate the threshold $\eta_{\mathscr{D}_{\Delta \varepsilon \Delta \varepsilon}}$

$$
\eta_{\mathscr{D}_{\Delta \varepsilon \Delta \varepsilon}}\left(f_{\theta}\right)=-4 \sigma^{2} \cdot \ln \alpha \cdot \sin \left(\pi f_{\theta} \Delta \theta\right)^{2}=-\ln \alpha \cdot \frac{T_{c}^{2}}{3} \cdot \sin \left(\pi f_{\theta} \Delta \theta\right)^{2} .
$$

Assuming the quantization noise has most influence over the speed measurement, IAS noise is stated to follow an uniform law in the same way as the quantization error, $\eta_{\mathscr{D} \tau \tau}\left(f_{\theta}\right)=\eta_{\mathscr{D}_{\Delta \varepsilon \Delta \varepsilon}}\left(f_{\theta}\right)$. It should be understood that these thresholds have not the same signification: 
- the quantization threshold $\eta_{\mathscr{D}_{\Delta \varepsilon \Delta \varepsilon}}\left(f_{\theta}\right)$ estimates, for every cyclic frequency, the amplitude separating the spectral components of a realisation $\mathscr{D}_{\Delta \varepsilon \Delta \varepsilon}\left(f_{\theta}\right)$ in two complementary groups of respective ratio $\alpha$ and $1-\alpha$. This assertion will be validated in the next paragraph,

- the quantization threshold $\eta_{\mathscr{D}_{\tau \tau}}\left(f_{\theta}\right)$ distinguishes the quantization noise perturbing the spectral observation of a realisation $\tau_{i}(\theta)$ from the real IAS variations experienced by the mechanism, under the probability $\alpha$.

The quantization threshold is formulated by the power spectral density of a time variation. However, the physical interpretation of a cyclic phenomenon is preferably done over angular speed variations. Eq. (A.10) can be used to scale the PSD of a time variation in $\mathrm{rad} / \mathrm{s}$, although the Fourier transform is not directly applied on the speed signal.

$$
\eta_{\mathscr{D}_{\omega \omega}}\left(f_{\theta}\right)=\frac{\omega_{0}^{4}}{\Delta \theta^{2}} \cdot \eta_{\mathscr{D}_{\tau \tau}\left(f_{\theta}\right)} .
$$

\subsection{Numerical validation}

The results are numerically put to the test accordingly to a statistical approach. A set containing 100 process realisations of random noise distributed according to a uniform distribution is numerically generated. Each realisation is made of $10^{5}$ points distributed over the interval $[0,1]$ and will be observed directly as the quantization error in simple form or processed by finite difference to simulate the quantization error in a finite difference form. The power spectral densities computed from each of these process realisations are respectively presented in Fig. 12a-b. The probability rate of different thresholds are defined in the left part of Table 3 . The numerical results are presented in the right part of the same table, through the empirical mean and variance of the rate of spectral components whose amplitude is higher than the threshold. For example, over the 100 process realisations, 10.09 percent of the spectral components obtained after finite differentiation of the uniform distribution are higher than the threshold defined using a probability rate of $10 \%$, which is coloured in green in Fig. 12. The statistical approach of the quantization noise estimation is numerically validated.
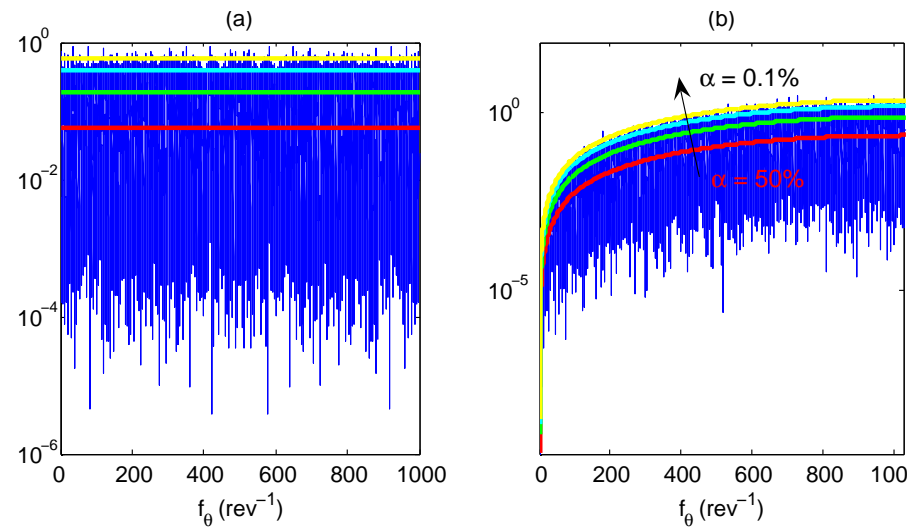

Figure 12: PSD of the quantization error when sutdied in $a$ ) a simple form and in $b$ ) a finite difference form.

\subsection{Experimental confirmation}

\subsubsection{Broad band perturbation}

The test rig presented in part 2.3.1 has been run under non stationary conditions. Since this paper considers the quantization perturbation, the experiment is tuned in order to enlighten this peculiar phenomenon in comparison to the geometric and electrical perturbations which are not considered. Ergo, measurement of the Elapsed Time is simultaneously performed by the help of two different clock frequencies of $105 \mathrm{kHz}$ and $20 \mathrm{kHz}$. These simultaneous measurements will allow to appraise the precision of the noise estimation for different set-up conditions where the quantization is insured to predominate both the geometrical error and the electrical noise. The two different counter clocks brought different rates of quantization error. 


\begin{tabular}{|c|c||c|c|c|c|}
\hline \multicolumn{2}{|c||}{ quantization threshold } & \multicolumn{4}{c|}{ Numerical results (in \%) } \\
\hline \multirow{2}{*}{$\begin{array}{c}\text { Color } \\
\text { (Fig. 12) }\end{array}$} & \multirow{2}{*}{$\alpha$ in \% } & \multicolumn{2}{c|}{$\begin{array}{c}\text { quantization Error } \\
\text { simple form }\end{array}$} & \multicolumn{2}{c|}{$\begin{array}{c}\text { quantization Error } \\
\text { finite difference }\end{array}$} \\
\cline { 3 - 6 } & & $\begin{array}{c}\text { Empirical } \\
\text { Mean }\end{array}$ & $\begin{array}{c}\text { Empirical } \\
\text { Variance }\end{array}$ & $\begin{array}{c}\text { Empirical } \\
\text { Mean }\end{array}$ & $\begin{array}{c}\text { Empirical } \\
\text { Variance }\end{array}$ \\
\hline Red & 50 & 49.97 & $3.43 \cdot 10^{-2}$ & 50.08 & $3.7 \cdot 10^{-2}$ \\
\hline Green & 10 & 10.00 & $1.43 \cdot 10^{-2}$ & 10.09 & $1.6 \cdot 10^{-2}$ \\
\hline Cyan & 1 & 1.00 & $1.13 \cdot 10^{-2}$ & 1.06 & $3.6 \cdot 10^{-3}$ \\
\hline Yellow & 0.1 & 0.10 & $1.75 \cdot 10^{-3}$ & 0.15 & $1.6 \cdot 10^{-3}$ \\
\hline
\end{tabular}

Table 3: Descriptions and results of the numerical simulations.

As it can be seen in Fig. 7, the experimental validation is made on a run up from 200 to $1000 \mathrm{rpm}$. For the acquisition to succeed, the counting process must produce at least 2 pulses between two consecutive encoder segments. Thus, the highest rotating speed sets a minimal value on the counting frequency or alternatively, a maximal value on the resolution: $F_{c}>R \cdot \omega / \pi$. Therefore, the encoder resolution has been artificially reduced to 512 pulses per revolution.
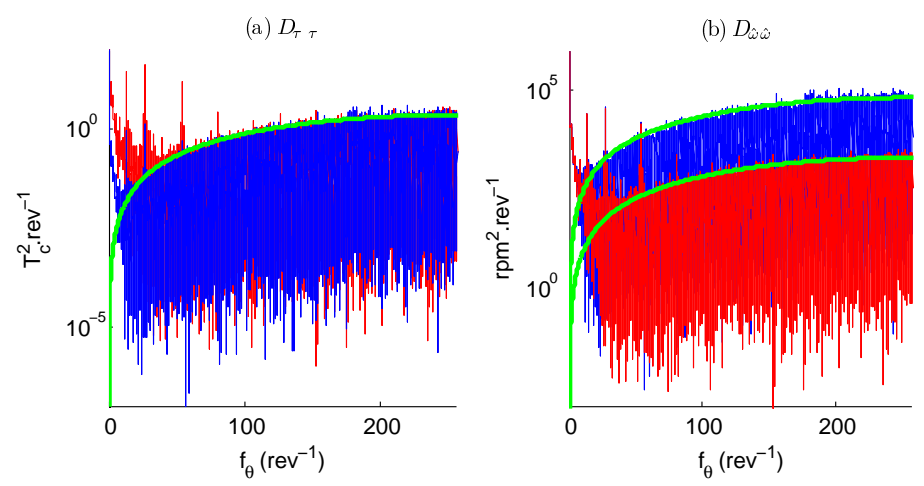

Figure 13: PSD of the measurements scaled $(a)$ in time and $(b)$ in speed. Counter frequency: $F_{c}=20 \mathrm{kHz}$ in blue, $F_{c}=105 \mathrm{kHz}$ in red. $\alpha=0.1 \%$

Power spectral densities obtained from each measurement are superposed in Fig. 13-a) with a quantization threshold obtained for $\alpha=0.1 \%$. To estimate the validity of the approach, the rates of spectral component whose amplitude are above the threshold have been measured over the interval $100 \mathrm{rev}^{-1}$ and $256 \mathrm{rev}^{-1}$. These rates are respectively equal to $0.103 \%\left(F_{c}=105 \mathrm{kHz}\right)$ and $0.059 \%\left(F_{c}=20 \mathrm{kHz}\right)$. Practically, this means that quantization noise is flooding the angular speed variations whose angular frequency is above $100 \mathrm{rev}^{-1}$ for both measurements. In Fig. 13 -b), both power spectral densities and quantization thresholds have been approximately translated into angular speed variations density using Eq. (23). This approximation enlightens the influence of the quantization noise over the angular speed variations. The angular periodic speed variations that are visible in the measurement made with the $20 \mathrm{kHz}$ counting frequency are always visible with the $105 \mathrm{kHz}$ frequency, and furthermore, they are very close in amplitude. For example, the amplitudes of the cyclic frequency components corresponding to the first three gear mesh harmonics between the output shaft and the axle shaft do not exceed $10 \%$ in relative difference (almost not visible on a logarithmic scale). Information within each signal may be different due to the difference in term of counting frequency, the threshold is well correlated with the experiments. The statistical approach is experimentally validated.

\subsubsection{Periodic perturbation}

As this phenomenon clearly appears only when speed is absolutely constant, these perturbations are more likely to occur in simulations than in actual measurements, and therefore are not studied here. 

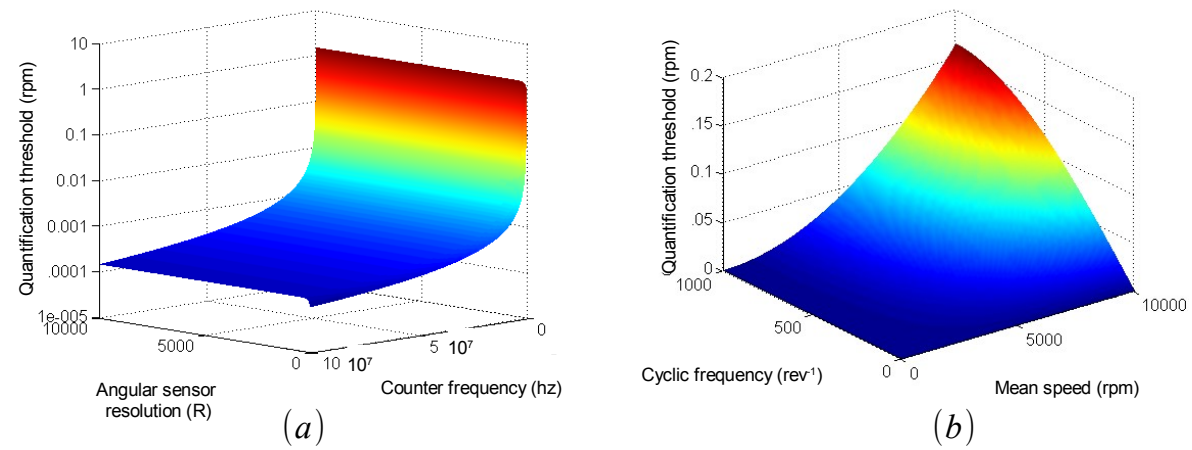

Figure 14: Evolution of quantization error $\eta_{\omega}$ (a) as a function of the tunable parameters and the environment parameters (b).

\subsection{Numerical application}

Once the statistical approach is validated numerically and experimentally, these results can be used to design an IAS monitoring system with regards to the cyclic phenomena expected in a peculiar mechanism. Indeed, the quantization threshold has been shown to depend on four different parameters, which can be classified within two groups:

- the cyclic frequency of a cyclic phenomena and the average speed during the measurement; these are the environment parameters and are dictated by the application itself,

- oppositely, the encoder resolution and the counting frequency can be tuned to reach a targeted noise level.

In Fig. 14 are plotted the evolution of the induced quantization noise as a function of the parameters of each group when the parameters of the other group are fixed. These figures directly result from the application of Eq.(22). The experimental results have shown the predominance of quantization noise amongst other kinds of perturbation listed in the introduction. However, the experiments have been run for relatively slow counting frequencies and this result has no claim to be general. Although some conditions are proven to enlighten quantization noise and have therefore been used to validate the work in the present paper, other conditions are expected to set other balance between the different sources of noise. This will be the subject of further works.

\section{Conclusion}

This paper has distinguished the IAS methodologies that yield a signal sampled in the time domain from those sampled in the angular domain. Because the study of mechanisms run under non stationary operating conditions is nowadays subject to an increasing attention, the latters need to be properly defined before an industrial development could be achieved. This paper focused on the Elapse Time measurement technique, and two major unawarenesses limiting this technique have been studied.

First, the aliasing process has been detailed. This phenomenon peculiarly limits the angular domain methodology based on a conventional angular sensor and is today impossible to be fully tackled since no analog filter can be successfully applied to the digital signal before it is sampled. However, its influence on the spectral observation has been thoroughly appraised and the following conclusions have been drawn:

- the measured spectrum is attenuated by a cardinal sine shape as compared to the spectrum of IAS,

- aliased phenomena are attenuated by more than $14 d B$ in the first quarter of the cyclic spectrum.

- it is possible to estimate the actual IAS variations of any cyclic phenomenon whose original cyclic frequency is known and subjected to aliasing in the case of low resolution encoder. 
Afterwards, the quantization phenomenon study has been conducted in two consecutive steps. The stationary case has been necessary to prove and to understand the influence of quantization on the spectral observation of the Elapse Time IAS signal. The spectral ghosts induced during these peculiar operation might not be observed in reality, except in case of very stationary mechanism or in case the counting clock is very slow.

Non-stationary operation generalization has been handled with the help of statistical tools. The quantization phenomenon becomes a spread noise and the signal to noise ratio has been accurately quantified on the spectral amplitude either scaled in time or in angular speed. Several parameters have been identified to have a precise impact on the quantization measurement, and the authors think this study will be of valuable help to efficiently design a IAS acquisition system based on the Elapse Time methodology depending on the characteristic of the mechanism operating parameters.

Some improvements are still to be done to improve IAS precision and general understanding. The geometrical errors of the tachometer gratings due to manufacturing process are still very difficult to dissociate from some mechanical phenomena of interest. The interpolation process incorporated in most of the angular sensors also introduces a cyclic pattern in the signal that might also hide synchronous phenomena. In the mean time, the IAS measurement process has uncertainties and thermal noise due to the conversion from mechanical motion into an electrical signal, the amplification of the sensor and the digitalisation of the signal, whose influence over the Elapse Time signal is not fully understood...

So many subjects that still need to be studied more deeply to appreciate the achievable performance of IAS signal in the wide and demanding fields of control and monitoring.

\section{References}

[1] Charles, P., Sinha, J. K., Gu, F., Lidstone, L., and Ball, A., 2009. "Detecting the crankshaft torsional vibration of diesel engines for combustion related diagnosis". Journal of Sound and Vibration, 321(3-5), pp. $1171-1185$.

[2] Renaudin, L., Bonnardot, F., Musy, O., Doray, J., and Rémond, D., 2010. "Natural roller bearing fault detection by angular measurement of true instantaneous angular speed". Mechanical Systems and Signal Processing, 24(7), pp. 1998 - 2011.

[3] Yuhua, L., Gu, F., Harris, G., Ball, A., Bennett, N., and Travis, K., 2005. "The measurement of instantaneous angular speed". Mechanical Systems and Signal Processing, 19(4), pp. 786-805.

[4] André, H., Bourdon, A., and Rémond, D., 2011. "On the use of the instantaneous angular speed measurement in non stationary mechanism monitoring.". Proceedings of the ASME 2011 International Design Engineering Technical Conferences \& 23rd biennal Conference on Mechanical Vibration and Noise IDETC/CIE2011, Washington, DC, USA.

[5] de León, F. C. G., and Pérez, P. A. M., 2010. "Discrete time interval measurement system: fundamentals, resolution and errors in the measurement of angular vibrations". Measurement Science and Technology, 21(7), p. 075101.

[6] Youssef, W., Guillet, F., and Elbadaoui, M., 2011. "Characterization of counter technique for instantaneous angular speed measurement: Application on gear box signal". Proceedings of the International Conference Surveillance 6, Université Technologique de Compiègne, France.

[7] Binder, J., 1992. "New generation of automotive sensors to fulfill the requirements of fuel economy and emission control". Sensors and Actuators A: Physical, 31(1-3), Mar., pp. 60-67.

[8] Halliwell, N., 1996. "The laser torsional vibrometer : A step forward in rotating machinery diagnostics". Journal of sound and vibration, 190(3), Jan., pp. 399-418.

[9] Li, X., and Meijer, G., 1998. "A novel low-cost noncontact resistive potentiometric sensor for the measurement of low speeds". IEEE Transactions on Instrumentation and Measurement, 47 (3)(3), pp. 776-781.

[10] Kaul, S., Koul, R., Bhat, C., Kaul, I., and Tickoo, A., 1997. "Use of a look-up table improves the accuracy of a low-cost resolver-based absolute shaft encoder". Measurement Science \& Technology, 8, pp. 329-331.

[11] Bertocco, M., Flammini, A., Marioli, D., and Taroni, A., 1999. "Robust and accurate real-time estimation of sensors signal parameters by a dsp approach". Instrumentation and Measurement Technology Conference, 1999. IMTC/99. Proceedings of the 16th IEEE, 2, pp. 697-702 vol.2.

[12] Gu, F., Yesilyurt, I., Yuhua, L., Harris, G., and Ball, A., 2006. "An investigation of the effects of measurement noise in the use of instantaneous angular speed for machine diagnosis". Mechanical Systems and Signal Processing, 20(6), Aug., pp. 1444-1460.

[13] Vakman, D., 2000. "New high precision frequency measurement". Measurement Science and Technology, 11(10), pp. $1493-1497$.

[14] Yu, S., and Zhang, X., 2010. "A data processing method for determining instantaneous angular speed and acceleration of crankshaft in an aircraft engine-propeller system using a magnetic encoder”. Mechanical Systems and Signal Processing, 24(4), pp. 1032 - 1048. 


\section{Appendix A. Theory of attenuation of IAS spectrum}

This appendix demonstrates the filtering effect due to Elapsed Time measurement. The first subsection details hypothesis of little variations, and the second one gives the correspondance between the actual IAS spectrum and the measured one. Quantization effect induced by the truncation of the Elapsed Time measurement is not taken into account.

Appendix A.1. Assumption on angular speed

The hypothesis is made that IAS is quite constant, so as to develop first order approximations. Then, it comes:

$$
\omega(\theta)=\omega_{0}+\delta \omega(\theta)
$$

with

$$
\left|\frac{\delta \omega(\theta)}{\omega_{0}}\right|<<1 \quad \forall \theta
$$

Using Eq.(1), it comes:

$$
d t=\frac{d \theta}{\omega_{0}+\delta \omega(\theta)} \approx \frac{1}{\omega_{0}} \cdot\left(1-\frac{\delta \omega(\theta)}{\omega_{0}}\right) \cdot d \theta
$$

Then, the time laps between two consecutive encoder pulses is noted as:

$$
\begin{gathered}
\tau(\theta)=\frac{1}{\omega_{0}} \cdot \int_{\Delta \theta}\left(1-\frac{\delta \omega(\theta)}{\omega_{0}}\right) \cdot d \theta \\
\tau(\theta)=\frac{1}{\omega_{0}} \cdot\left(\Delta \theta-\frac{[\delta \omega(\theta)]_{\Delta \theta}}{\omega_{0}}\right)
\end{gathered}
$$

with

$$
[\delta \omega(\theta)]_{\Delta \theta}=\int_{\Delta \theta} \delta \omega(\theta) \cdot d \theta \quad \text { and } \quad \frac{[\delta \omega(\theta)]_{\Delta \theta}}{\omega_{0}}<<\theta .
$$

The constant and variable parts of time laps are defined as:

$$
\tau_{0}=\frac{\Delta \theta}{\omega_{0}} \quad \delta t(\theta)=\frac{[\delta \omega(\theta)]_{\Delta \theta}}{\omega_{0}^{2}}
$$

and then :

$$
\tau(\theta)=\tau_{0}+\delta t(\theta)
$$

It is verified that :

$$
\left|\frac{\delta t(\theta)}{\tau_{0}}\right|<<1 \quad \forall \theta
$$

Appendix A.2. Effect of counting technique method

Regarding Eq. (2) and previous assumptions, it comes:

$$
\begin{gathered}
\bar{\omega}(\theta)=\frac{\Delta \theta}{\tau_{0}} \cdot \frac{1}{1+\frac{\delta t(\theta)}{\tau_{0}}} \approx \frac{\Delta \theta}{\tau_{0}} \cdot\left(1-\frac{\delta t(\theta)}{\tau_{0}}\right) \\
\bar{\omega}(\theta)=\omega_{0} \cdot\left(1-\frac{\tau(\theta)-\tau_{0}}{\tau_{0}}\right) \\
\bar{\omega}(\theta)=2 \cdot \omega_{0}-\frac{\omega_{0}^{2}}{\Delta \theta} \cdot \tau(\theta) .
\end{gathered}
$$


It is noted that in a first order approximation, the measured IAS is directly proportional to the elapsed time. Thus, measured IAS spectrum can be estimated directly through Elapsed Time method if the small angular speed variation is verified:

$$
\bar{\Omega}\left(f_{\theta}\right)=2 \cdot \omega_{0} \cdot \delta\left(f_{\theta}\right)-\frac{\omega_{0}^{2}}{\Delta \theta} \cdot \mathscr{T}\left(f_{\theta}\right) .
$$

For the end of demonstration, elapsed time will be replaced by its expression $\tau(\theta)=t(\theta)-t(\theta-\Delta \theta)$. Considering $T\left(f_{\theta}\right)$ the Fourier transform of $t(\theta)$, the amplitude spectrum in the cyclic frequency domain of the measured IAS at the first-order approximation is:

$$
\bar{\Omega}\left(f_{\theta}\right)=2 \cdot \omega_{0} \cdot \delta\left(f_{\theta}\right)-\frac{\omega_{0}^{2}}{\Delta \theta} \cdot T\left(f_{\theta}\right) \cdot\left(1-e^{-j 2 \pi f_{\theta} \Delta \theta}\right) .
$$

Furthermore, $T\left(f_{\theta}\right)$ can be found using Eq. (1) and first order approximation:

$$
t^{\prime}(\theta)=\frac{d t}{d \theta}(\theta)=\frac{1}{\omega(\theta)}=\frac{1}{\omega_{0}} \cdot\left(1-\frac{\delta \omega(\theta)}{\omega_{0}}\right)=\frac{1}{\omega_{0}} \cdot\left(2-\frac{\omega(\theta)}{\omega_{0}}\right) .
$$

Then, in the cyclic frequency domain:

$$
T\left(f_{\theta}\right)=\frac{1}{j 2 \pi f_{\theta}} \cdot \frac{1}{\omega_{0}^{2}} \cdot\left(2 \omega_{0} \cdot \delta\left(f_{\theta}\right)-\Omega\left(f_{\theta}\right)\right) .
$$

Using this last equation, the measured IAS can be expressed as a function of the actual IAS:

$$
\begin{gathered}
\bar{\Omega}\left(f_{\theta}\right)=2 \cdot \omega_{0} \cdot \delta\left(f_{\theta}\right)-\frac{\omega_{0}^{2}}{\Delta \theta} \cdot \frac{1}{j 2 \pi f_{\theta}} \cdot \frac{1}{\omega_{0}^{2}} \cdot\left(2 \cdot \omega_{0} \cdot \delta\left(f_{\theta}\right)-\Omega\left(f_{\theta}\right)\right) \cdot\left(1-e^{-j 2 \pi f_{\theta} \Delta \theta}\right) \\
\bar{\Omega}\left(f_{\theta}\right)=2 \cdot \omega_{0} \cdot \delta\left(f_{\theta}\right)-\left(2 \cdot \omega_{0} \cdot \delta\left(f_{\theta}\right)-\Omega\left(f_{\theta}\right)\right) \cdot e^{-j \pi f_{\theta} \Delta \theta} \cdot \frac{e^{j \pi f_{\theta} \Delta \theta}-e^{-j \pi f_{\theta} \Delta \theta}}{2 j \pi f_{\theta} \Delta \theta} \\
\bar{\Omega}\left(f_{\theta}\right)=2 \cdot \omega_{0} \cdot \delta\left(f_{\theta}\right)-\left(2 \cdot \omega_{0} \cdot \delta\left(f_{\theta}\right)-\Omega\left(f_{\theta}\right)\right) \cdot e^{-j \pi f_{\theta} \Delta \theta} \cdot s i n c_{\pi}\left(f_{\theta} \Delta \theta\right) .
\end{gathered}
$$

Finally, the spectrum of the first-order approximated measured angular speed can be simply expressed as:

$$
\bar{\Omega}\left(f_{\theta}\right)=\Omega\left(f_{\theta}\right) \cdot e^{-j \pi f_{\theta} \Delta \theta} \cdot \operatorname{sinc}_{\pi}\left(f_{\theta} \Delta \theta\right) .
$$

As a conclusion, it can be observed that, for an angular speed signal with negligible variations, the counting technique creates a low-pass filter with a $\sin c_{\pi}$ shape based on $\Delta \theta$ resolution of the encoder.

\section{Appendix B. Quantization precision}

Appendix B.1. periodic artefact induced by the stationary case

The real time separating the $i^{t h}$ rising edge and the counter start is defined such as:

$$
t_{i}=\frac{i p}{q} \cdot T_{c}+t_{0}
$$

As previously, quantization error is introduced through the measured time $\hat{t}\left(\theta_{i}\right)$,

$$
\hat{t}_{i}=\left\lceil\frac{i p}{q}+\frac{t_{0}}{T_{c}}\right\rceil \cdot T_{c},
$$

where $i \cdot p / q$ is not integer a priori. Therefore, the measured time between consecutive rising edges is given by:

$$
\hat{\tau}_{i}=\left(\left\lceil\frac{i p}{q}+\frac{t_{0}}{T_{c}}\right\rceil-\left\lceil\frac{(i-1) \cdot p}{q}+\frac{t_{0}}{T_{c}}\right\rceil\right) \cdot T_{c}
$$


Since the measured time can only equals an integer multiple of period $T_{c}$, there is $\forall i \in[|1 ; N|], \hat{\tau}\left(\theta_{i}\right) \neq \tau\left(\theta_{i}\right)$. Precisely, $\hat{\tau}\left(\theta_{i}\right)$ equals the integer values surrounding $\tau\left(\theta_{i}\right)$. Moreover, it is known that $\forall i \in \mathbb{N}, \exists !(n, r) \in \mathbb{N} \times[|0 ; q-1|] / i=$ $n \cdot q+r$. From Eq. (B.2), there is one and only one couple $(n, r) \in \mathbb{N} \times[|0 ; q-1|]$ such as

$$
\begin{aligned}
& \hat{t}\left(\theta_{i}\right)=\left\lceil\frac{(n q+r) \cdot p}{q}+\frac{t_{0}}{T_{c}}\right\rceil \cdot T_{c} \\
& \Longrightarrow \quad \hat{t}\left(\theta_{i}\right)=n t\left(\theta_{q}\right)+\hat{t}\left(\theta_{r}\right) \\
& \Longrightarrow \quad \hat{\tau}\left(\theta_{i}\right)=n t\left(\theta_{q}\right)+\hat{t}\left(\theta_{r}\right)-\left(n t\left(\theta_{q}\right)+\hat{t}\left(\theta_{r-1}\right)\right) \\
& \Longrightarrow \hat{\tau}\left(\theta_{i+n \cdot q}\right)=\hat{\tau}\left(\theta_{i}\right)
\end{aligned}
$$

The quantization phenomenon is proven to induce angle periodic variations of the IAS signal.

Appendix B.2. Quantization error in a finite difference form

The Wiener Khintchine Theorem implies that the power spectral density of the random signal $\Delta \varepsilon$ can be estimated from $\Delta E\left(f_{\theta}\right)$, the Fourier transform of one realisation $\Delta \varepsilon_{i}(\theta)$ by the relation:

$$
\mathscr{D}_{\Delta \varepsilon \Delta \varepsilon}\left(f_{\theta}\right) \triangleq \Delta E\left(f_{\theta}\right) \cdot \Delta E^{*}\left(f_{\theta}\right) .
$$

The Fourier transform being linear, the previous equation is associated to Eq. (20) to obtain:

$$
\mathscr{D}_{\Delta \varepsilon \Delta \varepsilon}\left(f_{\theta}\right) \triangleq\left(E\left(f_{\theta}\right)-E\left(f_{\theta}\right) \cdot e^{-2 j \pi f_{\theta} \Delta \theta}\right) \cdot\left(E\left(f_{\theta}\right)-E\left(f_{\theta}\right) \cdot e^{-2 j \pi f_{\theta} \Delta \theta}\right)^{*} .
$$

Knowing that $X^{*}\left(f_{\theta}\right)=\left(X\left(f_{\theta}\right)\right)^{*}$, we get:

$$
\mathscr{D}_{\Delta \varepsilon \Delta \varepsilon}\left(f_{\theta}\right) \triangleq\left(E\left(f_{\theta}\right) \cdot E\left(f_{\theta}\right)^{*}\right) \cdot\left(2-e^{-2 j \pi f_{\theta} \Delta \theta}-e^{2 j \pi f_{\theta} \Delta \theta}\right),
$$

with $\sin (x)^{2}=\left(\frac{e^{j x}-e^{-j x}}{-2 j}\right)^{2}$, we get:

$$
\mathscr{D}_{\Delta \varepsilon \Delta \varepsilon}\left(f_{\theta}\right) \triangleq\left(E\left(f_{\theta}\right) \cdot E\left(f_{\theta}\right)^{*}\right) \cdot\left(2 \cdot \sin \left(\pi f_{\theta} \Delta \theta\right)\right)^{2} .
$$

Using once again Wiener Khintchine theorem,

$$
\mathscr{D}_{\Delta \varepsilon \Delta \varepsilon}\left(f_{\theta}\right) \triangleq \mathscr{D}_{\varepsilon \varepsilon}\left(f_{\theta}\right) \cdot\left(2 \cdot \sin \left(\pi f_{\theta} \Delta \theta\right)^{2}\right)^{2} .
$$

